\title{
An Approach to Measuring Step Excrescence Effects in the Presence of a Pressure Gradient
}

\author{
Anne M. Bender ${ }^{1}$, J._Russell Elliott ${ }^{2}$, Yuto Shinagawa ${ }^{3}$, Andrea J. Korntheuer ${ }^{4}$, and Aaron Drake $^{5}$ \\ Northrop Grumman Corporation, El Segundo, CA 90245 \\ Russell V. Westphal ${ }^{6}$ \\ California Polytechnic State University, San Luis Obispo, CA 93407 \\ Sergiy Gerashchenko ${ }^{7}$ and Beverley J. McKeon ${ }^{8}$ \\ California Institute of Technology, Pasadena, CA 91125 \\ Shuya Yoshioka ${ }^{9}$ \\ Ritsumeikan University, Shiga, Japan
}

\begin{abstract}
An experimental study was undertaken to determine the effects of step excrescences on boundary layer transition using a unique ground test facility in which the test model was propelled though still air. The models used were designed to have a nominally constant pressure gradient so that the results would be relevant to laminar flow aircraft whose wings often have long runs of mildly favorable pressure gradient. The models had an integrated continuously adjustable twodimensional step, which could be adjusted to be forward-facing or aft-facing. The large model was used to increase the Reynolds numbers examined so that the results are applicable to laminar flow flight vehicles. Multiple measurement methods, including Preston tubes, hot wires, accelerometers, a boundary layer traverse, and static pressure taps were used to provide comparison data, and to add to the physical understanding of the results. The propelled-model test approach required that the instrumentation be self-contained and ride along with the model as the carrier vehicle moved down the test track. Due to the relatively short times available for data-taking (approximately 15-30 seconds per run), the initialization and data analysis techniques had to be tailored for this application.
\end{abstract}

\section{Nomenclature}

$C_{p} \quad=$ pressure coefficient

${ }^{1}$ Aerodynamics Engineer, Aerodynamic Design and Analysis.

${ }^{2}$ Aerodynamics Engineer, Flight Analysis.

${ }^{3}$ Aerodynamics Engineer, Aerodynamic Design and Analysis.

${ }^{4}$ Aerodynamics Engineer, Aerodynamic Design and Analysis;..

${ }^{5}$ Manager, Flight Analysis. AIAA Senior Member.

${ }^{6}$ Professor, Mechanical Engineering. AIAA Senior Member.

${ }^{7}$ Postdoctoral scholar. Current affiliation: Cornell University.

${ }^{8}$ Associate Professor of Aeronautics, Graduate Aerospace Laboratories (GALCIT). AIAA Member.

${ }^{9}$ Associate Professor, Mechanical Engineering. 


$\begin{array}{ll}C f & =\text { skin friction coefficient } \\ k & =\text { excrescence height } \\ K & =\text { dimensionless pressure gradient parameter } \\ R e_{k} & =\text { dimensionless excrescence height } \\ R e_{t r} & =\text { dimensionless transition location } \\ R e_{x k} & =\text { dimensionless location of excrescence } \\ S & =\text { surface distance } \\ \mathrm{U} & =\text { freestream velocity } \\ u_{k} & =\text { velocity at height } \mathrm{k} \\ X & =\text { chordwise distance }\end{array}$

\section{Introduction}

WORTHROP Grumman has been examining the manufacturability concerns associated with designing 1 and building a laminar flow aircraft for several years. These investigations began with a low-speed study in a small wind tunnel, the purpose of which was to examine the effect of pressure gradient on the allowable excrescence height. The results of the initial study were promising - indicating that favorable pressure gradient had a significant stabilizing effect and offered the potential for loosening of manufacturing tolerances. However, this initial study was limited to low chord and transition Reynolds numbers $(<1$ million) by the constraints of the wind tunnel facility. As such, the results of the study were not directly applicable to aircraft of interest. The objective of the current study was to extend the results to higher transition Reynolds numbers so that the results would be directly applicable to systems of interest.

Already at the limit of the low-speed wind tunnel's capabilities, it was necessary to find a new facility with a low disturbance environment and a higher Reynolds number capability. Tohoku University's Towing Wind Tunnel was identified as a potential facility in August 2005 and subsequent proof-of-concept testing indicated that it was a viable candidate for higher Reynolds number excrescence testing. The Towing Wind Tunnel Facility (TWT) is a unique propelled-modeled facility located in Hyuga, Japan.

The testing conducted for the current study, called the Surface Excrescence Transition Study (SETS), was designed around the capabilities of the Towing Wind Tunnel. New models were designed and fabricated to take advantage of the large physical size of the facility. The instrumentation was also optimized for the TWT in order to exploit the high Reynolds number capability and to extend the relations developed for transition Reynolds number $\left(\mathrm{Re}_{\mathrm{tr}}\right)$ as a function of excrescence height Reynolds number $\left(\operatorname{Re}_{\mathrm{k}}\right)$ to over $4 \times 10^{6}$ in the current study.

\section{Model Design and Fabrication}

\section{A. Model Design}

The objective of the model aerodynamic design was to create models that preserved near-constant chordwise and spanwise dimensionless pressure gradient $(K)$ distributions. The specific values of $K$ of the two models were chosen after examining the nondimensional pressure gradients of long-endurance, laminar flow Intelligence, Surveillance and Reconnaissance (ISR) aircraft. The chosen values of $K$ bracket the most-applicable range of pressure gradient values for relevant aircraft at these Reynolds numbers. The target $K$ values were $0.2 \times 10^{-7}$ (Gradient-Zero) and $0.5 \times 10^{-7}$ (Gradient-A).

The initial design for the favorable pressure gradient model (Gradient-A) was done using XFLR5, a panel-code coupled with a two-equation lagged dissipation integral boundary layer formulation. While this method is relatively inaccurate, it provided a way to quickly manipulate the geometry and make rapid $C_{p}$ calculations in order to acquire a rough outer mold line (OML) shape for the model. The OML was then used to create a 2-D Navier-Stokes model using Gridgen. This grid was then run through a 2-D/3-D RANS

flow solver called CFL3D. Based upon the computational fluid dynamics (CFD) results $s_{2}$ the curvature of the OML was further manipulated using a NURBS modeling package called Rhino. This process was repeated for each model until an OML that provided the desired pressure distribution was found.

Because of the low aspect ratio of the model (resulting from the large chord in order to take advantage of the large size of the carrier vehicle to increase the Reynolds number), splitter plates were used to maximize the two-dimensional character of the flow. Figures 1 and 2 show a cross-section of the OML 
lines for the models, as well as the planform of the splitter plates. To size the splitter plates a 3-D NavierStokes model was created in Gridgen and analyzed with CFL3D. An iterative process was used to size the splitter plates, increasing the lateral extent of these surfaces progressively until the spanwise uniformity of the pressure was achieved within 5\% (see Figure 3).

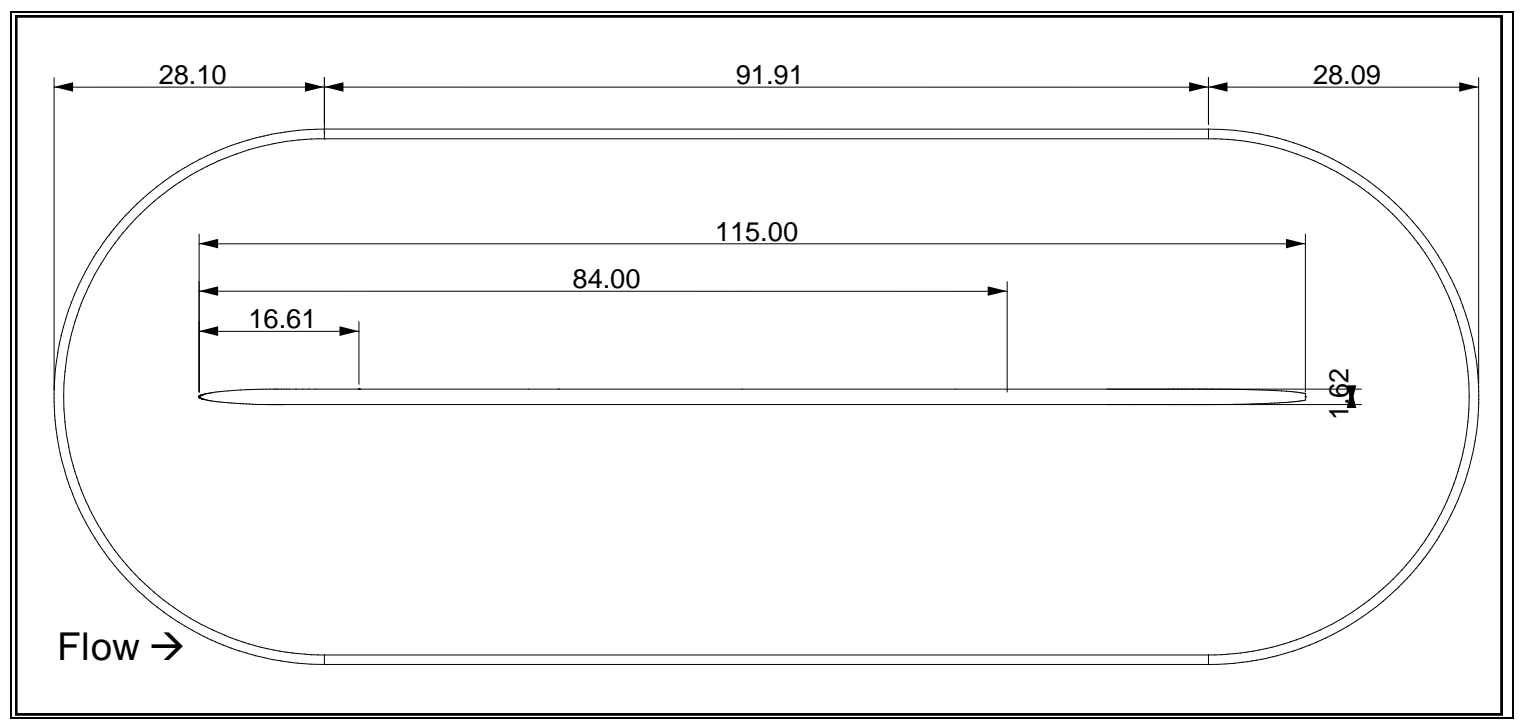

Figure 1. Top view of Gradient-Zero model showing splitter plate (dimensions in inches).

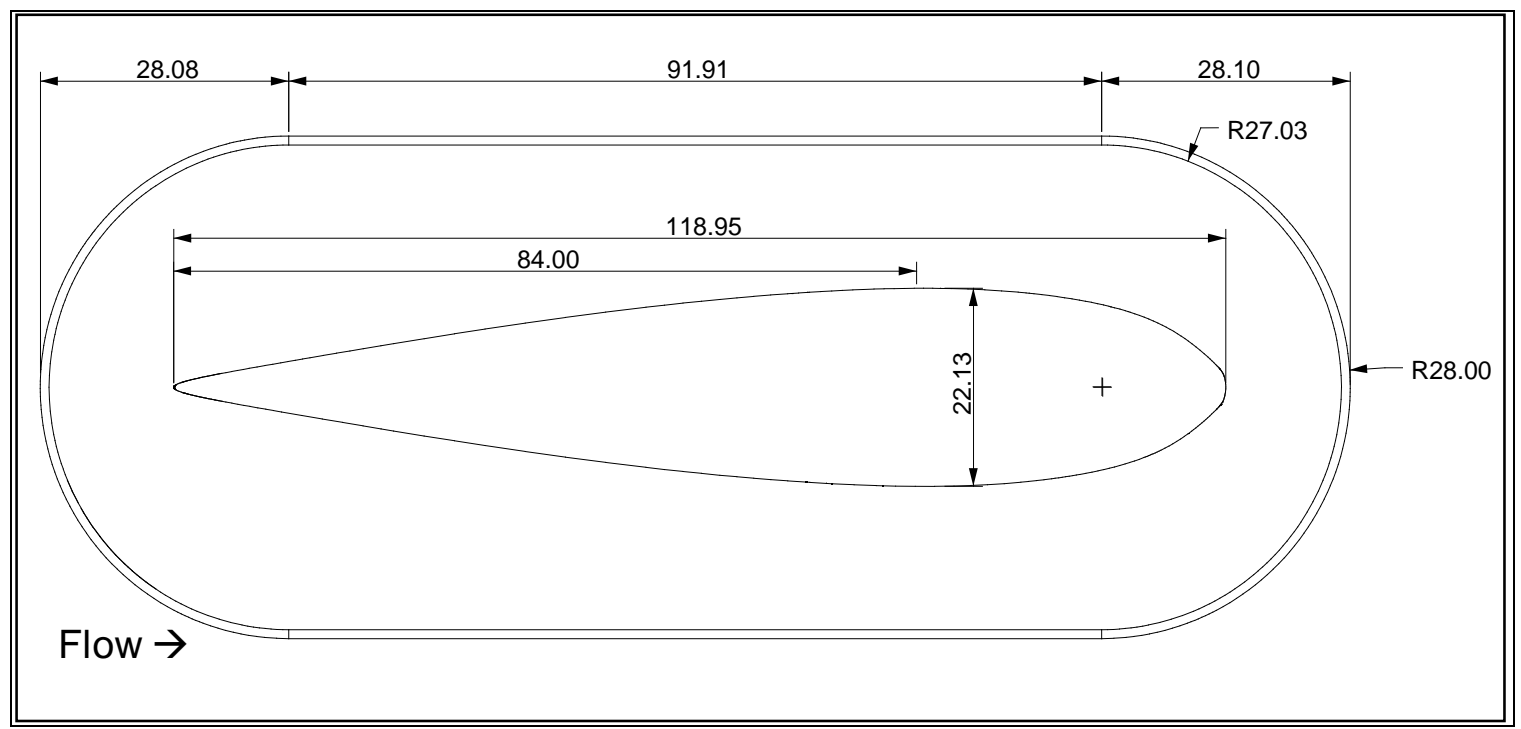

Figure 2. Top view of Gradient-A model showing splitter plate (dimensions in inches). 


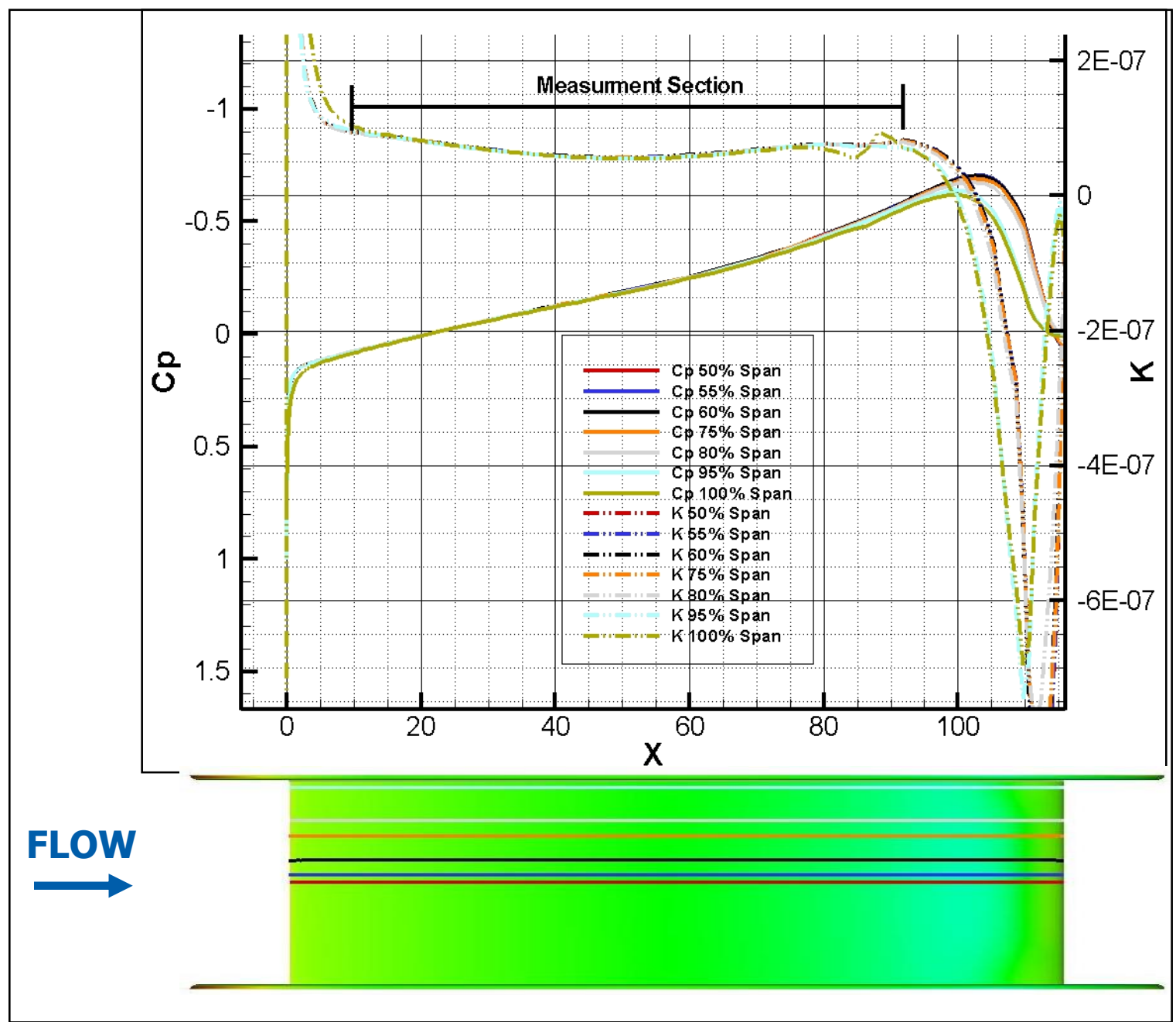

Figure 3. Free air spanwise and chordwise pressure gradient of Gradient-A model.

\section{B. Modeling of Unsteady Effects}

Early in the model design it was evident that to attain the desired pressure distributions the Gradient-A model would have a very broad aft end, creating a bluff body shape. Because the testing was to take place at low dynamic pressures, concerns arose regarding the stability of the flow separating off the aft end of the model. In order to investigate whether the model exhibited significant vortex shedding, a 2-D timeaccurate model for the Gradient-A model was created.

The time-accurate flow solver CFL3D was used for the unsteady flow analysis. In order to minimize analysis time and computational resources, the unsteady analysis was performed in 2-D. The 2-D analysis uses a Detached Eddy Simulation (DES) coupled with a Spalart-Alamaras (SA) turbulence model. This 2$\mathrm{D}$ unsteady analysis is very conservative in that it tends to overpredict unsteadiness.

The resulting temporal lift variation was examined and the frequency of the vortex shedding was found to be less than that of the Tolmien-Schlichting waves and therefore unlikely to induce early transition. However, because the model did exhibit low frequency vortex shedding, it was necessary to analyze how much the leading edge stagnation point moved and if this would also affect the transition location of the model.

Figure 4 (below) shows the center of the stagnation point and maximum stagnation point travel due to the vortex shedding. It was seen the stagnation point travel due to the vortex shedding is acceptable and will not induce early separation. The maximum travel of the stagnation point using the conservative analysis was no more than 0.03 inches. It was then concluded that the vortex shedding had negligible effect upon the models and therefore no further action was required to modify the model design. 


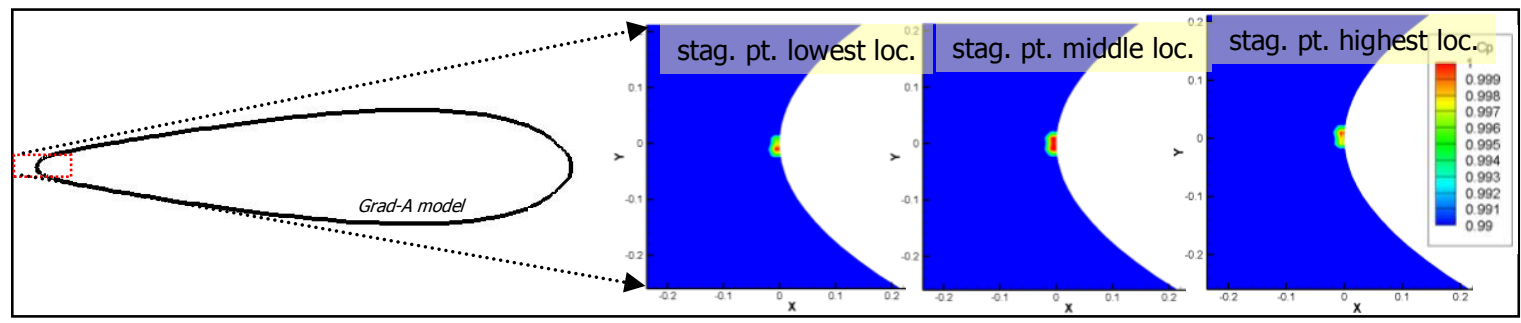

Figure 4. Maximum movement of stagnation point as indicated by conservative unsteady analysis.

\section{Model Fabrication}

The excrescences under investigation in the study are purely two-dimensional steps, both forward- and aft-facing. Thus it was critical that the model be able to create a true 2-D step excrescence, without rampup or ramp-down, and that step remain perpendicular to the surface, as the angle of the step excrescence can alter the boundary layer stability.

The models' leading edge assemblies incorporated a sub-assembly with 3 precision linear translating slides to move the leading edge assembly laterally such that 0.001 " incremental step heights could be created. The step excrescence that was created was the same for the entire 4-foot vertical length of the leading edge assembly. The leading edge assembly was prevented from "racking" relative to the rest of the model by side tie-plates, which supported the weight of the model while the step assembly was allowed to move smoothly. Aft of the leading edge assembly there was a midsection assembly; the model leading edge moved with respect to this section. Aft of the midsection is the remaining OML of the model, which was a fiberglass skin with interior reinforcements to maintain shape.

The majority of model components were CNC machined from CAD models. The leading edge and midsection assemblies were made from aluminum-6061. The leading edge pieces were manufactured from a single solid aluminum billet in order to avoid unintended steps and gaps. The area surrounding the step location was stainless steel to ensure the tolerance, perpendicularity, and rigidity of the step excrescence. The side tie plates, which supported the weight of the model as steps were dialed in, were also stainless steel.

The measurement side and non-measurement side skins were a fiberglass layup. The skins on the nonmeasurement side include access panels for instrumentation and step adjustment. The measurement side skins have no access panels so as not to disturb the boundary layer being measured.

A Laser Tracker inspection of the fiberglass skin panels showed the worst-case OML variation was less than 0.010 inches over the entire approximate 4 by 7 foot area. The maximum OML variation was seen at the edges of the model at the interface of the splitter plate, which is outside the measurement region. Significantly tighter tolerances were held throughout the measurement region.

\section{Instrumentation Approach}

The instrumentation needed to measure the location of transition and important flow properties, as well as fulfilling some requirements unique to the facility. Because the facility is a propelled-model facility, all of the instrumentation needed to be self-contained with regards to power and memory, as well as be able to physically fit on, and ride along with, the carrier vehicle. Since, the time the carrier vehicle is in the test section is relatively short - on the order of 15-30 seconds - and the transient time before and after is comparatively large, the measurement methods needed to be able to accommodate these inherent conditions.

The measurement methods selected for these experiments were intended to complement each other and provide overlapping sources of information on the boundary layer. Multiple measurement methods also aid in comparing results to other work in the literature, as typically only one measurement method is considered primary in any one experiment, yet the results can be different depending on the method.

The parameters measured during the test included pressure coefficient distributions, skin friction 
coefficients, boundary layer velocity profiles and disturbance frequency distributions. Static pressure taps were used to measure the surface pressure distributions on the models. A boundary layer traverse was used to measure boundary layer velocity profiles at the step location in order to reduce step heights to dimensionless $R e_{k}$ values. Preston tubes were used to the measure skin friction distributions as well as to bound the transition location. Hot wire anemometry and accelerometers were used to measure the dynamic flow environment. The instrumentation setup and results from each of the measurement methods are presented in Section 5.

The integration of instrumentation and models was checked in two separate shakedown wind tunnel tests in Northrop Grumman's 7x10 foot wind tunnel in Hawthorne, CA. The shakedown tests were also used to confirm the CFD-predicted pressure distributions and the absence of undesirable unsteady effects. Data analysis methods were also refined during these tests.

\section{Test Facility}

The Towing Wind Tunnel facility ${ }^{1}$ was built in 2003 and is owned and operated by Tohoku University. The facility is located in Hyuga, Miyazaki Prefecture, Japan. This newly developed facility consists of a carrier vehicle called the "HART vehicle" on which the test article is placed, a guideway in which the HART vehicle runs, and a control system. The facility was constructed on a former MAGLEV testing track owned by Railway Technical Research Institute of Japan ${ }^{2}$.

The track is $3.46 \mathrm{~m}$ wide and $6.9 \mathrm{~km}$ long. However, only the first $1900 \mathrm{~m}$ of the total $6.9 \mathrm{~km}$ length track is currently used by the Towing Wind Tunnel. A diagram of the facility is shown in Figure 5. The first $910 \mathrm{~m}$ of the track is the acceleration section, the next $515 \mathrm{~m}$ is the test section and the final $475 \mathrm{~m}$ is the deceleration section. The entire test section is covered by a hood to shield it from winds, but in Figure 5 , for visual aid, the hood that covers the second half of this test section is not shown.

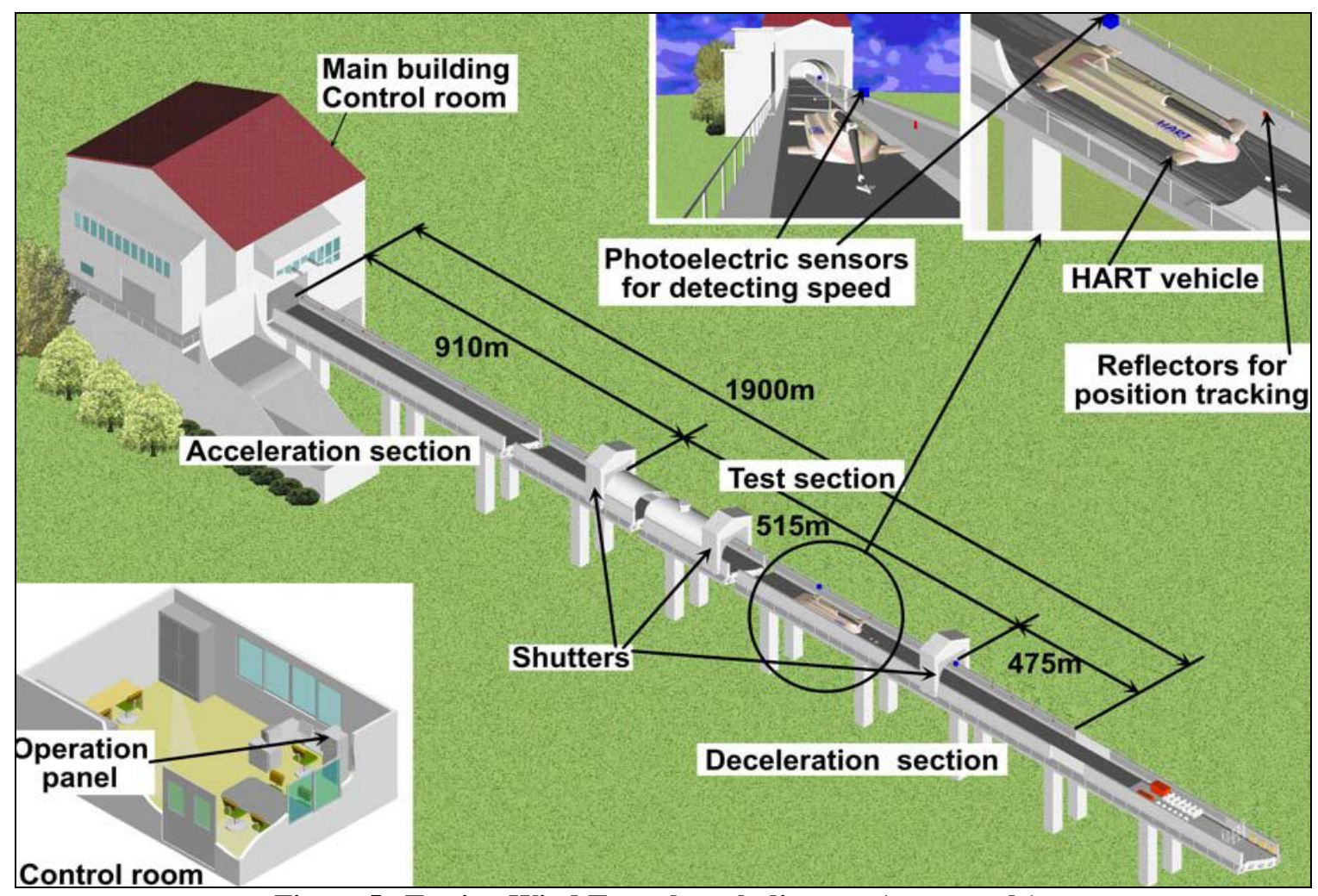

Figure 5. Towing Wind Tunnel track diagram (not to scale).

The HART vehicle has eight tires underneath its body and four tires along its side. Four out of the eight underbody tires are driven by in-wheel $55 \mathrm{~kW}$ electric motors, powered by onboard lithium-ion batteries. 
The side tires are forced to contact the guide walls by springs so that the HART vehicle keeps its position in the center of the track (Figure 6).

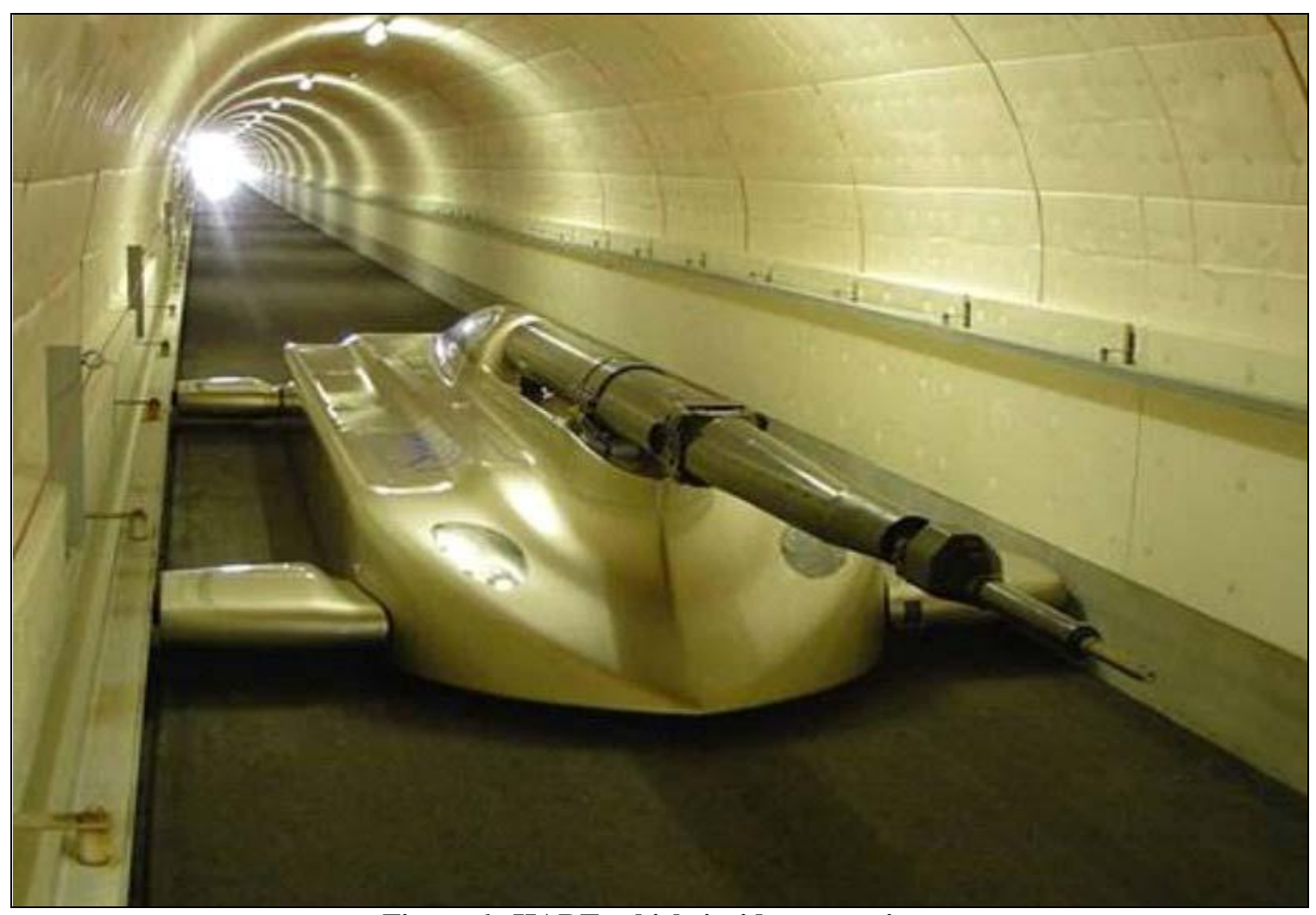

Figure 6. HART vehicle inside test section.

The HART vehicle is equipped with a hydraulically-actuated support arm to minimize vibrations experienced by the models. The maximum speed of the HART vehicle is $30 \mathrm{~m} / \mathrm{s}$ during manned operation and $50 \mathrm{~m} / \mathrm{s}$ during unmanned operation (clear tunnel). Currently, the only mode of operation of the HART vehicle is manned operation.

The HART vehicle is controlled by setting its ground speed. The air speed is slightly slower as compared with the ground speed because the HART vehicle entrains flow as it moves through the test section. At the end of the test section, the air speed is slower than the ground speed by roughly $10 \%$. This difference could be minimized in the future by accelerating the HART vehicle while in the test section, though this modification was not available for the tests reported here. However, this variation of airspeed did not adversely affect the SETS experiments because the SETS data is analyzed over a small subset of the time that the HART vehicle is in the test section. During this short period of time, the conditions can be considered to be constant. The pressure signal in the Preston tubes and other pressure-based instrumentation settles in approximately 1-2 seconds, while the hot wire data is instantaneous. For the present experiments, the data from the entire time the HART vehicle was in the test section was analyzed and checked for constancy of results, however, only a subset of that data was used in the final processing of the results. Thus, the slow decay of airspeed is not a factor and the speed of the run was taken to be the speed at the time that the data was analyzed.

\section{Instrumentation and Results}

\section{A. Static Taps and Pressure System}

Two chordwise rows of static pressure taps and one row of spanwise taps were installed on the models to measure - and check the uniformity of - the pressure distributions. The chordwise static pressure tap 
distribution was denser near the leading edge and spaced more coarsely towards the aft end of the models. Two pressure taps per chordwise row were located near the leading edge on the non-measurement side of the model, in order to help assess the location of the stagnation point. The pressure taps were 0.020 " holes drilled in the surface with 0.063 " stainless steel hard lines bonded beneath, which connected to Tygon tubing. The Tygon tubing was routed inside the model and was accessible through access ports from the non-measurement side of the model. The tubing exited the model at the aft end of the non-measurement side of the model.

A pressure system was procured specifically for the testing. This pressure system needed to record pressures from the 60+ static pressure taps and up to 18 Preston tubes simultaneously, in addition to a Pitot probe. The components of the pressure system included a Scanivalve DSM 3400 and four ZOC22/TCU units, which were mounted to the underside of the model lower splitter plate. During the test, three ZOCs were installed on the carrier vehicle and the remaining ZOC was treated as a spare. The range of the transducers was $+/-10$ in $\mathrm{H}_{2} \mathrm{O}$ and the DSM 3400 logged 2 scans/second on all 96 channels simultaneously and saved the data on its internal memory which was downloaded to a laptop following each run. The pressure system was powered by two 12-volt motorcycle batteries connected in series to provide 24-volts. The measured static pressure distributions for Gradient-Zero and Gradient-A are shown in Figure 7.

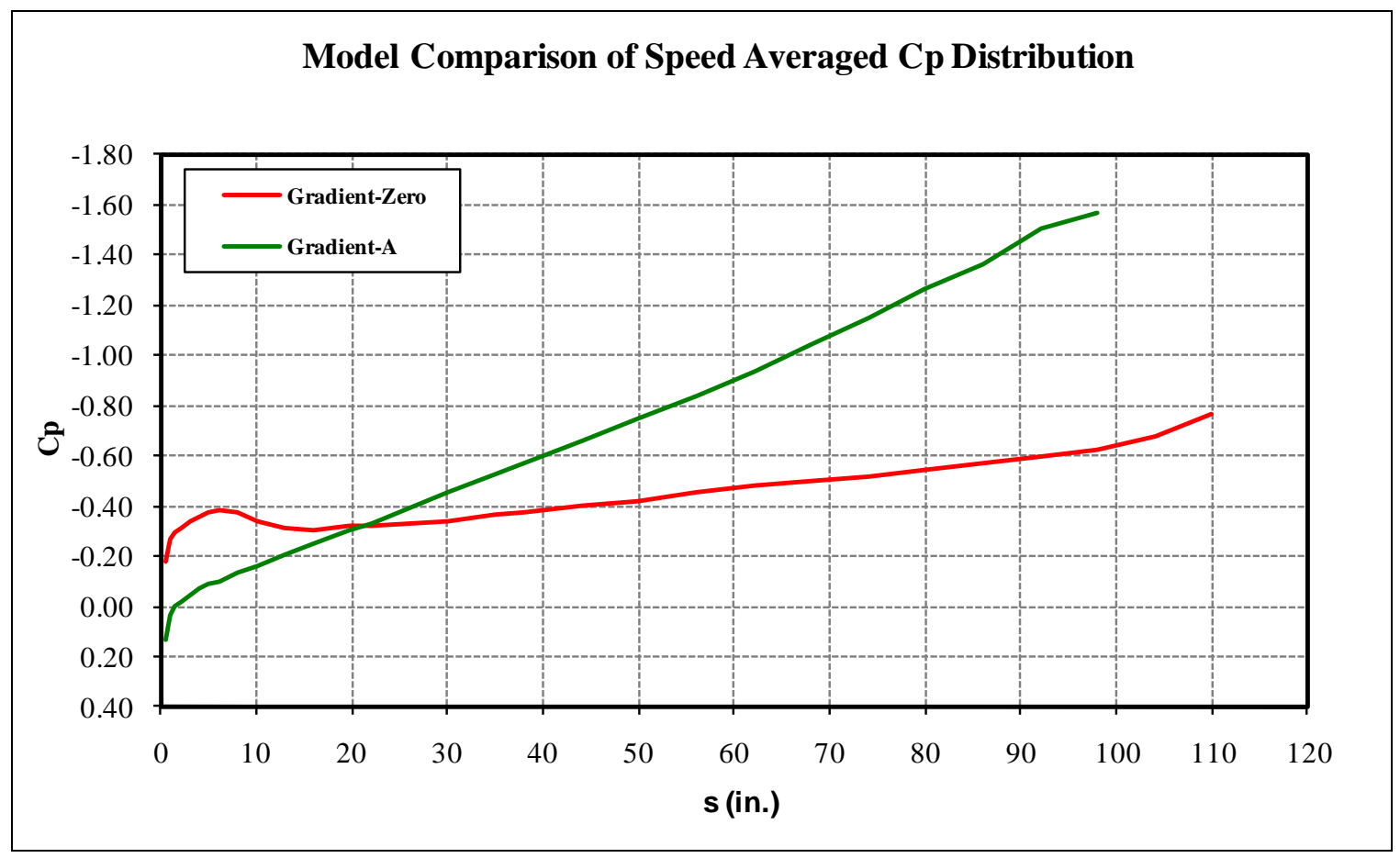

Figure 7. Model comparison of speed averaged Cp distribution.

\section{B. Boundary Layer Traverse}

A traverse was developed that was capable of positioning either a total pressure tube or a hot wire probe through the boundary layer. The traversing system, called Boundary Layer Data System (BLDS), is similar to boundary layer velocity profile measurement devices that have been developed previously ${ }^{3,4,5}$ for flight test use. The BLDS device records time-stamped position, local dynamic pressure, static pressure and temperature data at each point. The BLDS attaches to the models with double-sided structural adhesive tape, and thus can be placed at any position on the models. It is entirely self-contained, with its own battery power and on-board memory. A serial cable connection with a laptop computer was used to initiate the devices prior to a run, and to download data after each run.

A light-sensitive trigger was designed to start the data collection on the BLDS device once the HART vehicle moved into the test section. The test section is covered by a hood and thus the ambient light transitions from outside, ambient light to relative darkness (shadow), once the HART vehicle enters the hooded test section. The light trigger used a photoresistor $(\mathrm{CdS})$ and provided analog light level and 
digital trigger signals, which were also recorded.

The BLDS device was used to measure local velocity profiles at the step location on each of the models. The local velocity profiles were needed in order to reduce the dimensioned step heights into dimensionless $R e_{k}$ values. A sample measured boundary layer profile is shown in Figure 8. All of the boundary layer profiles measured on Gradient-A are shown in Figure 9. Different colors represent different runs at different speeds. Because the necessary parameter for the $R e_{k}$ calculation is a dimensioned velocity, namely $u_{k}$, the final form of the velocity profiles for this study, as shown in the figures, was $k$ in inches and $U$ in meters per second.

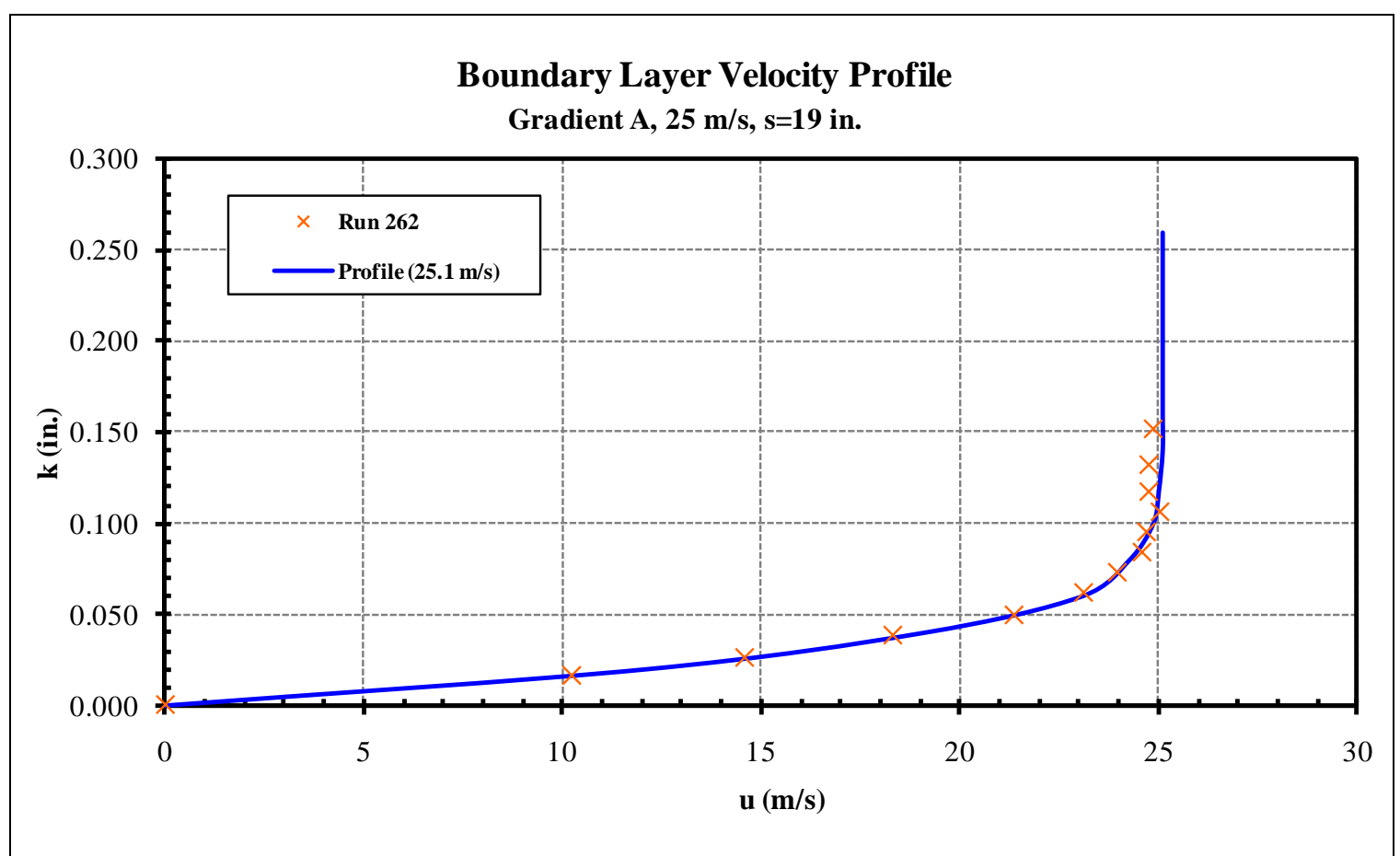

Figure 8. Gradient-A boundary layer velocity profile at $25 \mathrm{~m} / \mathrm{s}$. 


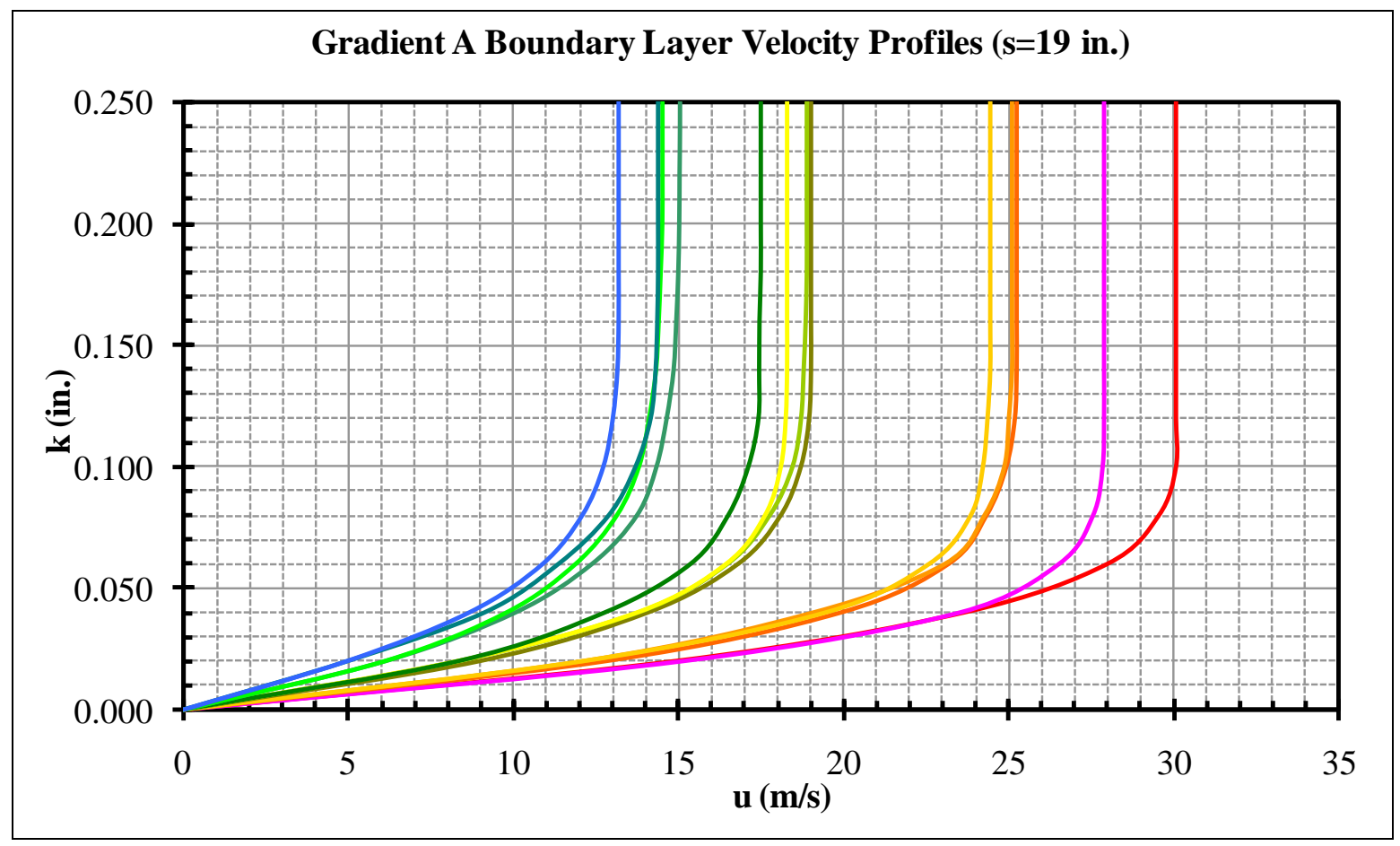

Figure 9. Gradient-A boundary layer velocity profile summary.

\section{Preston Tubes}

Preston tubes were used to measure skin friction distributions on the models. The necessary surface static pressure for a Preston tube skin friction measurement was obtained by interpolating the data from the static taps. Poll's calibration ${ }^{6}$ was used for laminar flow and Patel's calibration ${ }^{7}$ was used for turbulent flow. Preston tubes with outside diameters of 0.020 ", 0.025 " and 0.032 ", each with a ratio of inside diameter to outside diameter of 0.6 , were used depending on boundary layer thickness.

Pre-test predictions of skin friction coefficient and boundary layer thickness were calculated using a parabolic boundary layer program called $\mathrm{IBL}^{8}$. IBL computes 2D laminar, transitional, or turbulent boundary layers with prescribed distributions of surface pressure, heat transfer, and/or mass transfer. IBL outputs boundary layer properties such as the displacement thickness, momentum thickness, boundary layer thickness, and velocity profiles, as a function of streamwise distance. The IBL results were used to help set expectations of relative skin friction levels and to size the Preston tubes.

The Preston tubes were attached with the same double-sided structural adhesive that was used to attach the BLDS traverse. Preston tubes were mounted on the models nominally along a 15-degree half-angle line extending from the point where the leading edge meets the splitter plate, on both upper and lower surface (see Figure 10). This configuration of Preston tubes was the standard configuration used, though other arrangements were sometimes used. Thus there were typically rows of Preston tubes that varied in both spanwise and chordwise locations. Each row consisted of 7 Preston tubes, with the last centerline Preston tube being shared by both rows. 


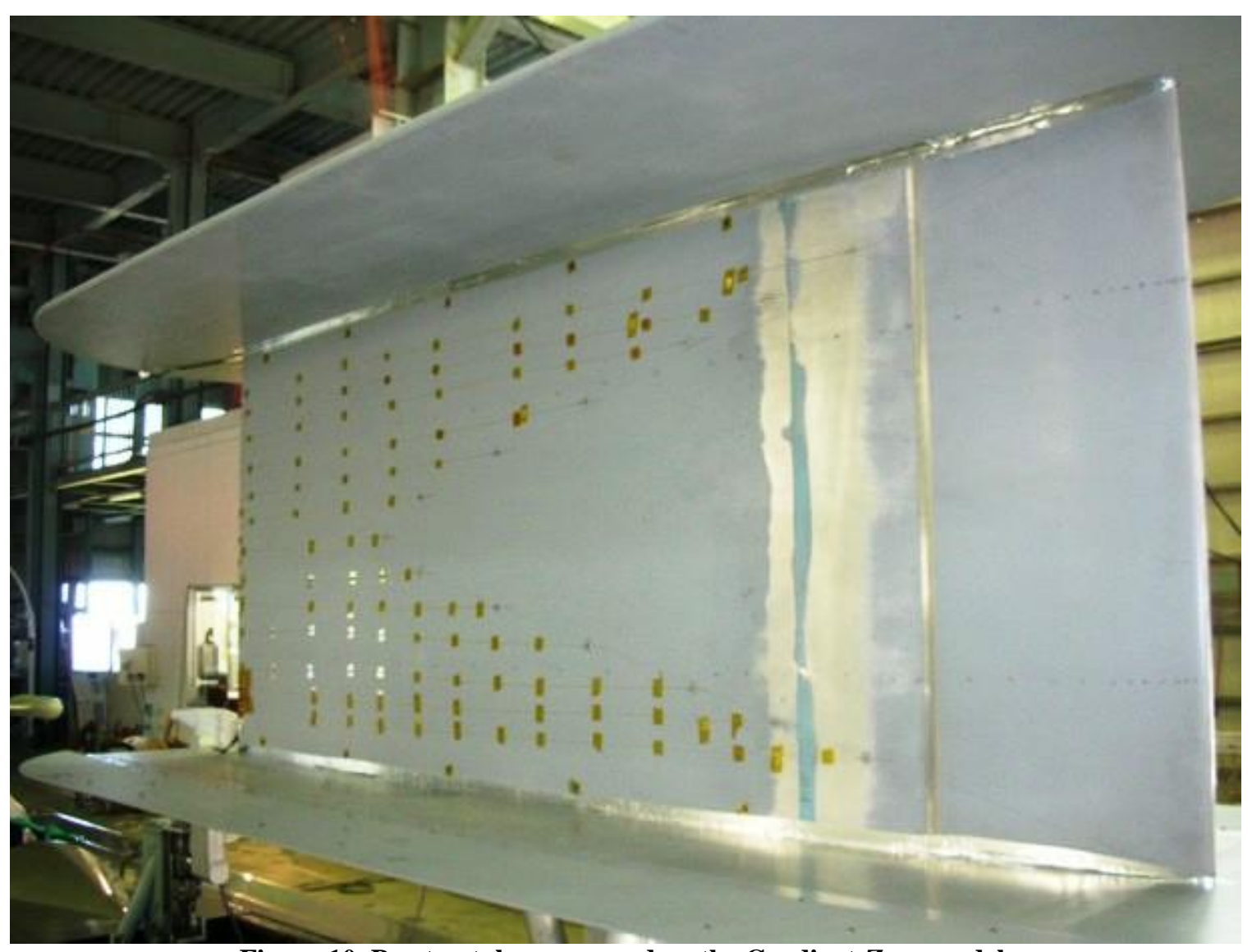

Figure 10. Preston tubes arranged on the Gradient-Zero model.

Skin friction distributions as measured by the Preston tubes are shown in Figures 11-12. The first figure is the clean plate (no excrescence) skin friction distribution for the Gradient-A model at a freestream speed of $28 \mathrm{~m} / \mathrm{s}$. The plot shows the skin friction coefficient results as measured by both the inboard and outboard row of Preston tubes. The plot also has IBL-predicted dashed reference lines for both fully laminar and turbulent-at-the-step boundary layers. These dashed reference lines serve only as quick check of the expected relative levels of laminar versus turbulent flow, and are not used in the data reduction otherwise. Figure 12 again shows the measured skin friction distribution for Gradient-A, but now at a reduced speed $(16 \mathrm{~m} / \mathrm{s})$ and with a forward-step excrescence of dimensionless height $R e_{k}=1886$. The clean plate skin friction distribution for Gradient-A (for the comparable freestream speed) is shown in Figure 12 as a solid green line. The rise of skin friction coefficient as compared to this measured clean plate skin friction distribution (the solid green line) is the basis for determination of transition for the Preston tube measurements. 


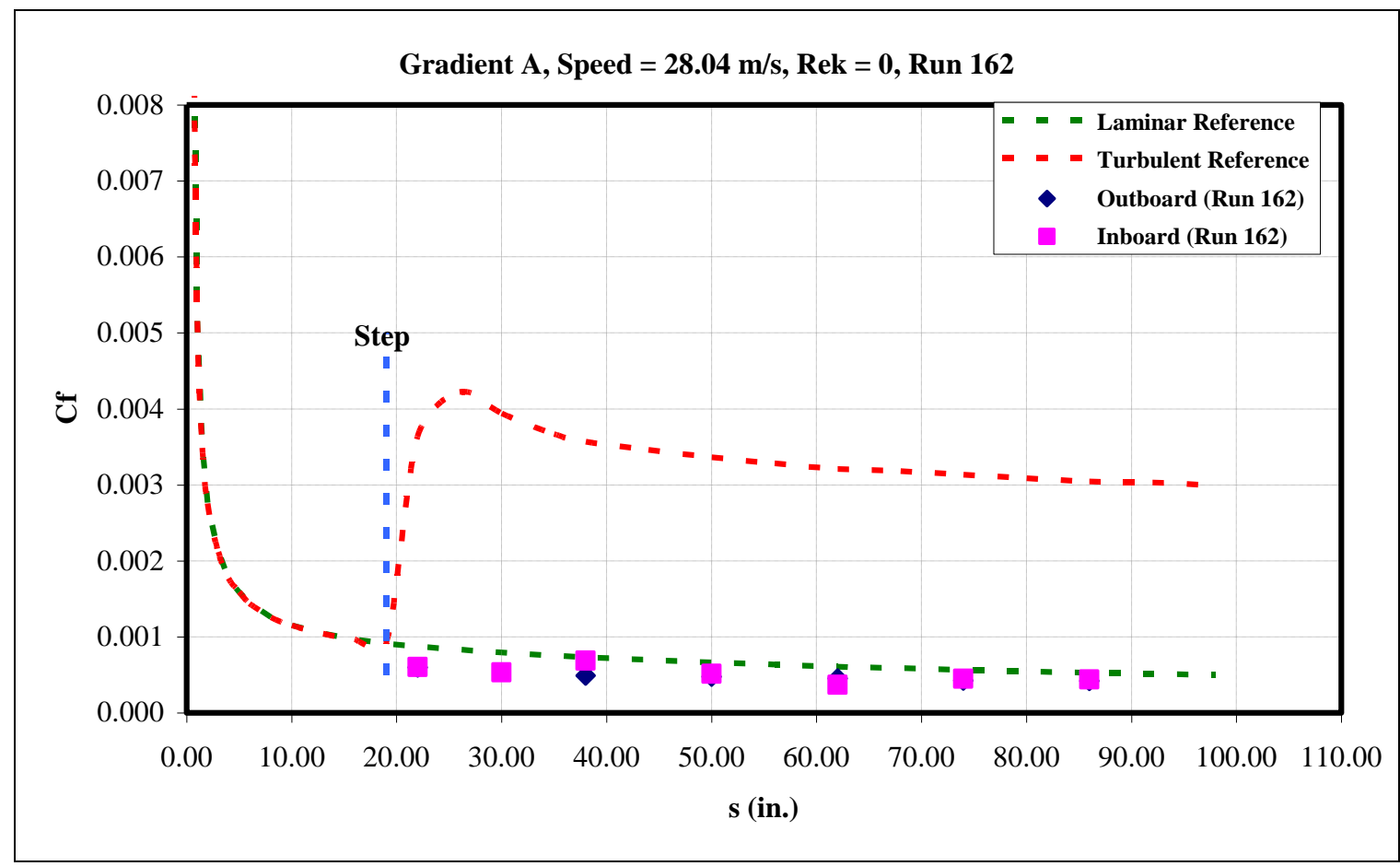

Figure 11. Clean plate skin friction distribution measured by Preston tubes.

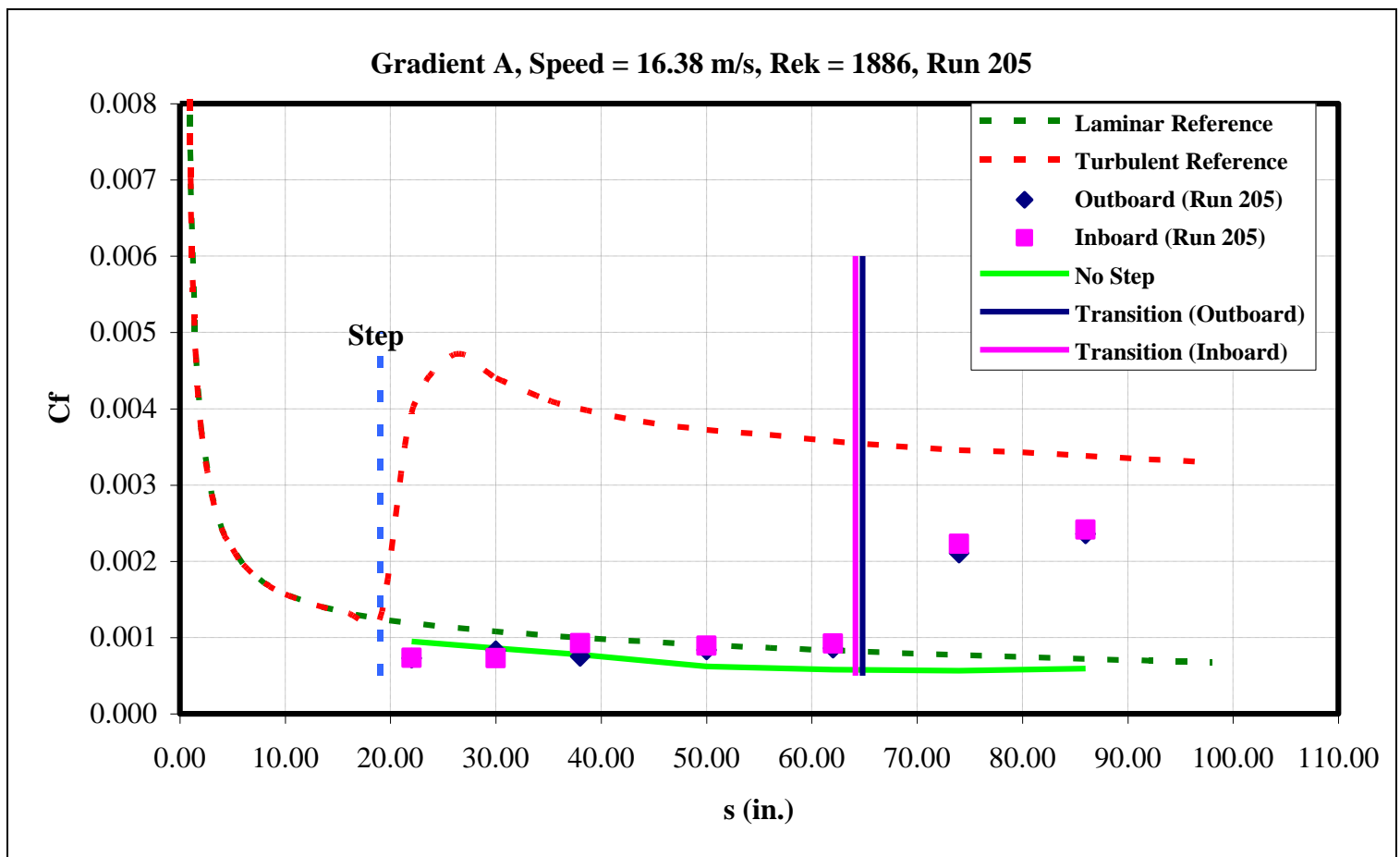

Figure 12. 0.070-in forward-facing step excrescence skin friction distribution measured by Preston tubes.

\section{Hot-wires and Accelerometers}

A standard four-channel constant temperature hot-wire anemometer (AA Lab Systems AN-1005) was used for flow velocity measurements. The hot-wires were Dantec single gold-plated boundary layer probes 
\#55P05. A Nikon D300 digital camera with a Tamron SPAF $180 \mathrm{~mm}$ lens was used to determine the height of the hot-wire above the surface within the pixel accuracy of about 50-100 microns. The hot-wires were calibrated separately from the HART carrier vehicle using a calibration jet.

Two accelerometers were adhered to the surface to record two components of model vibrations because vibrations could be a source of flow fluctuations. Each accelerometer had its own power supply with internal rechargeable battery, which worked simultaneously as an amplifier with adjustable gain.

The flow velocity, model vibrations and temperature fluctuations measured during the testing were recorded using a laptop computer together with a National Instruments data acquisition card.

The data taken by the hot-wires and accelerometers is discussed in detail in the companion paper AIAA-2010-374 . The data includes the experimental results on velocity power spectrum evolution and turbulence intensity measurements. Comparisons are also made between the Preston tube and hot-wire results.

In general, the hot-wires indicated an earlier onset of transition than the Preston tubes. The velocity spectral broadening and degree of intermittency (and RMS) seen by the hot-wire is more sensitive to disturbances than is the mean velocity profile. The data indicated that only after the velocity profile itself changed form from laminar to turbulent, was the increase in skin friction coefficient measured by the Preston tubes such that transition was flagged.

\section{E. Excrescence Relations}

Excrescence relations were developed from the Preston tube skin friction results. Each run produced a single transition location for the given setup. The excrescence relations developed are plots of dimensionless step height $\left(R e_{k}\right)$ versus transition Reynolds number $\left(R e_{t r}\right)$, or, versus the transition Reynolds number minus the dimensionless step location $\left(R e_{t r}-R e_{x k}\right)$. The relationship is specific to the pressure gradient and step geometry (e.g. forward- or aft-facing step). An example of the excrescence relation is shown in Figure 13, in this case, for aft-facing steps with $K=0.2 \times 10^{-7}$ (Gradient-Zero model).

The closed symbols on the plot represent measured transition locations. The open symbols represent runs wherein transition was not measured on the plate (i.e. the flow was all laminar for that condition). The transition Reynolds number value of the open symbols is shown at the end of the measurement section of the model - transition is at least greater than that $R e_{t r}$ value. The complete set of excrescence relations are shown in full in the companion paper AIAA-2010-375 ${ }^{10}$. In general, the excrescence relation results show a very stabilizing effect of pressure gradient, suggesting that manufacturing tolerances can be loosened in areas of even mildly favorable pressure gradient. As has been demonstrated in other work, the aft-facing steps are found to be more destabilizing than forward-facing steps. The companion paper discusses the results as well as the applicability to laminar flow aircraft manufacturing. 


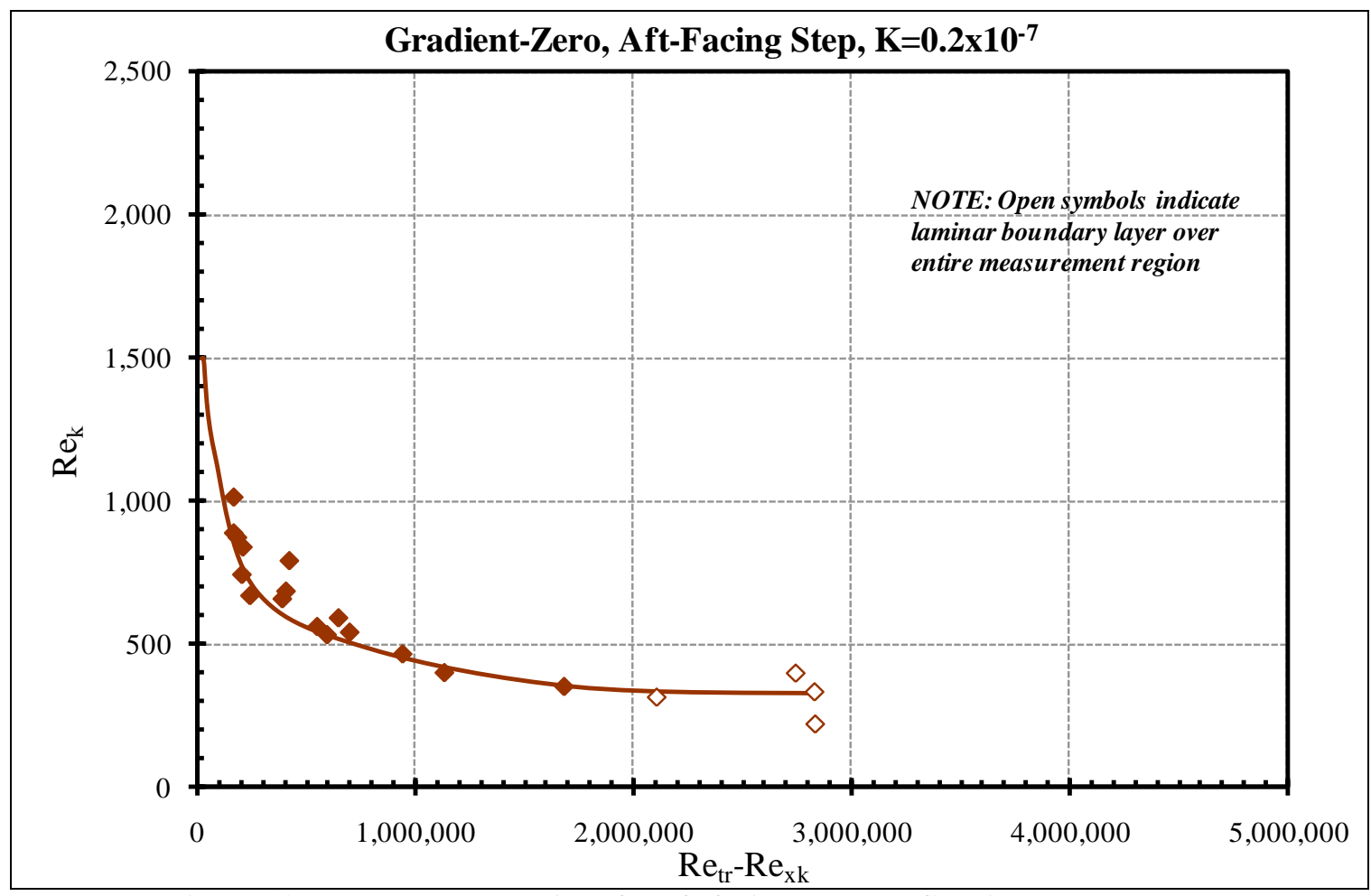

Figure 13. Excrescence relations for aft-facing steps on Gradient-Zero model.

\section{Conclusions}

A unique model-propelled test facility has been used with surface pressure, Preston tube, Pitot profiles, and hot-wire flow measurements to study the influence of step excrescences on transition. This approach enabled the use of physically large models so that transition Reynolds numbers up to 4 million could be achieved. The Preston tubes proved to be the easiest and most reliable way to locate transition because over a dozen Preston tubes could easily be used simultaneously whereas attempting to take as many measurements with hot-wires would have added significant time and complexity to the experiment. Because of the short on-condition times inherent to the propelled-model facility, simultaneous measurements at multiple locations was important.

The comparison and appreciation of the differences between the hot-wire measurements (which tended to indicate transition first) and the Preston tube measurements (which indicated transition later, only after the mean profile was modified, as confirmed by the traversing data) was perhaps the most interesting result from the experiment. However, the primary objective of the experiments was to develop excrescence relations (as further discussed in the companion paper AIAA-2010-375), which was accomplished with the experimental approach described herein.

\section{Acknowledgments}

This work was sponsored by the U.S. Air Force Research Laboratory, Air Vehicles Directorate.

The contributions of Mr. Donald Frame and Mr. Steven Jordan to the development of the boundary layer data system are gratefully acknowledged. 


\section{References}

${ }^{1}$ Yoshioka, Kikuchi, Ohta, Kato, Song, and Kohama, "Measurement of ground effect and boundary-layer transition by towing wind tunnel," 2009 Fluid Dyn. Res. 41021408 (12pp).

${ }^{2}$ Takahashi, K., "The outline of MLU002N on Miyazaki test track," Int. Conf. on Speedup Technology for Railway and MAGLEV Vehicles vol 1 pp 329-33, 1993.

${ }^{3}$ Westphal, Bleazard, Drake, Bender, et al, “A Compact, Self-Contained System for Boundary Layer Measurement in Flight", AIAA 2006-3828.

${ }^{4}$ Westphal, R. V., Frame, D., Jordan, S., Wanner Jr., A., Thompson, B., Bender, A., and Drake, A., "Design of a Third-Generation Boundary Layer Measurement System," AIAA-2008-7332.

${ }^{5}$ Bender, Drake, Westphal, et al., "Development and Flight Demonstration of Self-Contained Boundary Layer Measurement Devices ," AIAA-2008-7333.

${ }^{6}$ Poll, P.I.A., "A Note on the Use of Surface Pitot Tubes for the Measurement of Skin Friction in Laminar Boundary Layers", College of Aeronautics Report 8307, Cranfield Institute of Technology, Cranfield, Bedford, U.K., March 1983.

${ }^{7}$ Patel, V.C., "Calibration of the Preston Tube and Limitations on its Use in Pressure Gradients", Engineering Laboratory, Cambridge University, December 21, 1964.

${ }^{8}$ Murphy, J. D. and Davies, C.A., Users Guide - Ames Inlet Boundary Layer Program MK I. NASA TM X62211, January 1973.

${ }^{9}$ Gerashchenko S., McKeon B.J., Westphal R.V., Bender A.M., Drake, A., "Hot-wire Measurements of the Influence of Surface Steps on Transition in Favorable Pressure Gradient Boundary Layers", Paper 2010-374, 48 AIAA Aerospace Sciences Meeting, 2010.

${ }^{10}$ Drake A., Bender A.M., Korntheuer A., Westphal R.V., McKeon B.J., Gerashchenko S., Rohe W., and Dale G., "Step Excrescence Effects for Manufacturing Tolerances on Laminar Flow Wings", Paper 2010-375, 48 ${ }^{\text {th }}$ AIAA Aerospace Sciences Meeting, 2010. 